\title{
Protein Expression Analysis by Immunohistochemistry
}

National Cancer Institute

\section{Source}

National Cancer Institute. Protein Expression Analysis by Immunohistochemistry. NCI

Thesaurus. Code C162597.

The use of immunohistochemical methods to detect the expression of proteins in a sample. 